\title{
El modelo matemático de Fourier para el calentamiento terrestre
}

Felícitas Morales Álvarez ${ }^{1}$

\section{Resumen}

En este documento se presenta una articulación teórica del modelo matemático que representa la distribución de temperaturas en una esfera sólida, desarrollado en la teoría analítica del calor por J. Fourier, con la intención subyacente del autor de explicar el calentamiento terrestre debido a los efectos solares. Para lograrlo hacemos un análisis epistemológico de las hipótesis usadas y su traducción al lenguaje matemático con condiciones de frontera en el problema específico de determinar la ecuación de distribución de temperaturas en el sólido esférico.

Palabras Clave:Epistemología, distribución de temperaturas, conducción de calor.

PACS 01.40.-d, 01.50.-i, 01.65. +g, 91.35.Dc.

1 Tecnológico de Estudios Superiores de Cuautitlán Izcalli, México. 


\section{Abstract}

This paper presents a theoretical articulation of the mathematical model representing the temperature distribution in a solid sphere, developed in the heat analytical theory of J. Fourier, with the author underlying intention to explain the warming due to solar effects. To do this, we carry out an epistemological analysis of the assumptions used and their translation to mathematical language with boundary conditions on the specific problem of determining the temperature distribution equation in spherical solid.

Keywords: Epistemology, temperature distribution, heat conduction.

PACS 01.40.-d, 01.50.-i, 01.65. +g, 91.35.Dc. 


\section{Introducción}

La visión sistémica llamada socioepistemología en matemática educativa ha permitido explicar fenómenos didácticos en términos de cómo se enseña, cómo se aprende y qué se aprende. En este tenor, Farfán (1997) reportó una investigación que buscó significar el concepto de convergencia de series infinitas, entre profesores universitarios, dando entre otros resultados una caracterización del pensamiento matemático subyacente en la teoría del calor, donde se establece que, para la época, estaba suficientemente incorporado el paradigma del pensamiento físico, los principios matemáticos del equilibrio de los cuerpos y de los fluidos establecidos desde la antigüedad clásica por Arquímedes, las leyes de los cuerpos graves establecidas por Galileo y, sobre éstas, las leyes que rigen el sistema del mundo debidas a Newton.

El problema reportado por Farfán (1997) consistió en describir el comportamiento del fenómeno de propagación de calor, buscando para ello lo estable y permanente en el fluir del tiempo, es decir, planteó en su hipótesis inicial como indispensable la significación que da origen al conocimiento matemático en juego, es decir, la determinación del estado estacionario. Sin embargo, concluyó la autora, este concepto físico no es producto de la primera experiencia sensible; se encontró que su abstracción presentaba una tarea cognitiva muy compleja no propicia para recrearse en el aula. A partir de este resultado recomienda no centrar la atención en los conceptos y sus diferentes estructuraciones de forma aislada, sino tratar con las prácticas que producen o favorecen la necesidad de dichos conceptos. (Cantoral y Farfán, 2003)

La lectura de este estudio dio paso a otra investigación, donde Morales (2003) centró la atención en un escrito de J. B. Fourier (1827) acerca de las temperaturas terrestres, presentado como una primera aplicación práctica de la teoría analítica del calor. El objetivo de esta tesis fue el de observar la modelación matemática del problema de calentamiento terrestre y rescatar significados e intenciones de dicha actividad. Como resultados que pueden subrayarse en este estudio resaltan que:

El modelo de calentamiento terrestre de Fourier fue concebido previamente a la escritura de la Teoría analítica del calor. Dicho modelo consiste en el análisis de las tres fuentes principales del calor terrestre, una de ellas es que nuestro planeta participa de una temperatura común de los espacios planetarios, debido a la situación de nuestro sistema solar, donde todos los puntos de esa región del universo comparten una temperatura constante.

Una segunda fuente será el calor primitivo o calor de origen de la tierra, es decir, la tierra en un principio tenía una cierta temperatura muy elevada y fue enfriándose poco a poco desde la superficie, de manera que ahora sólo está caliente en su interior.

La tercera fuente continua de calor es el sol, cuyos efectos divide en dos categorías; efectos periódicos debidos al día y la noche y efectos constantes, los 
cuales se observan a una profundidad superior a los treinta metros por debajo de la superficie y es el resultado de la acción permanente de los rayos solares y la forma desigual en la que se exponen las partes de la superficie, desde el ecuador hasta los polos. En esta última causa, Fourier se encontró con el problema de explicar otros aspectos a considerar, tal como la distribución uniforme del calor debido a la atmósfera y al agua del planeta. De esta manera, concibe al planeta como una esfera sólida para fines prácticos, pero establece la ecuación de conducción de calor considerando únicamente la distribución de temperaturas en la superficie y su interacción con el medio ambiente; en este documento presentaremos la articulación de dicho modelo matemático con el fenómeno físico que le da origen.

\section{Perspectiva teórica}

La disciplina desde la que nace el presente estudio es la matemática educativa, disciplina que estudia la construcción del conocimiento matemático y su desarrollo en el sistema didáctico, aceptando como objetos de estudio los procesos de enseñanza aprendizaje de los contenidos matemáticos en situación escolar; es así cómo son diversas las problemáticas que se abordan y también diversos los enfoques teóricos que existen en torno a ella. La socioepistemología es uno de tales enfoques y el marco desde el cual se desarrollará el presente estudio.

Una de las principales contribuciones de la aproximación socioepistemológica, ha sido la de nutrir a la reflexión matemática y apoyar la didáctica, con base en la construcción social e individual del conocimiento, buscando una investigación sistémica de los fenómenos de producción, adquisición y difusión del conocimiento desde una perspectiva que incorpore las cuatro componentes fundamentales en la construcción del conocimiento: su naturaleza epistemológica, su dimensión sociocultural, los planos de lo cognitivo y los mecanismos de institucionalización vía la enseñanza. (Cantoral y Farfán, 2003)

La dimensión socioepistemológica. La epistemología estudia la naturaleza y validez del conocimiento, con el propósito de hacer un estudio crítico del desarrollo, métodos y resultados de las ciencias. La dimensión epistemológica incorporada en nuestra aproximación, se basa en la teoría de la epistemología genética desarrollada por J. Piaget, que se propuso encarar la reconstrucción del modo en que reproducen los conocimientos apelando al estudio de los procedimientos efectivos y el lenguaje científico, así como a la indagación histórica. (Albert, 1999)

Dicha teoría presentó una propuesta de la evolución del conocimiento: el conocimiento pasaría de un estado a otro de equilibrio a través de un desequilibrio de transición, en el curso del cual las relaciones consideradas por el sujeto en el estado anterior estarían en contradicción, ya sea por la consideración de relaciones 
nuevas o por la tendencia a coordinarlas. Esta fase de conflicto sería superada durante una fase de reorganización y de coordinación que llevaría a un nuevo estado de equilibrio. Aplicar esta teoría al conocimiento matemático deviene en que la epistemología juegue un papel protagónico en la disciplina.

De esta manera, la epistemología de un conocimiento interviene en la investigación a un nivel general, ya que se asume que el fenómeno educativo no es simplemente la transmisión de conocimientos; sino que este concierne a una cultura, no se limita a integrar asuntos referentes a la naturaleza histórica, sino en construir los distintos contextos teóricos que permitan involucrar los fenómenos didácticos encontrados, así como su incorporación efectiva en la enseñanza (Farfán, 1999). Bajo esa consideración será el análisis epistemológico el que responderá cuáles son los procesos generales del pensamiento que gobiernan el conocimiento.

La aproximación socioepistemológica ha puesto de manifiesto que la matemática escolar en el sistema didáctico y particularmente en la educación superior está al servicio de otras ciencias y por lo tanto de otras prácticas de referencia de donde, a su vez, adquiere sentido y significado. Es así como el análisis de los procesos de construcción del conocimiento matemático en un contexto físico es el eje que guía la presente investigación.

\section{Ecuación de conducción de calor}

Para los fines de este trabajo nos interesa analizar no sólo el cómo, sino también la intención subyacente con la que se estableció la ecuación de conducción de calor de la esfera, para poder lograr esto debemos revisar no únicamente el trabajo final terminado, sino rescatar asimismo los comentarios, justificaciones, explicaciones y postulaciones que el autor, en este caso Fourier, nos plantea al mismo tiempo que desarrolla su producción, para poder acceder a estas intenciones es que se hace necesario revisar la obra original, y explicarla con sus correspondientes acotaciones.

En Fourier, (1827) ${ }^{\dagger}$ Fourier establece la ecuación de distribución de temperaturas para su modelo de la siguiente forma: Considera un sólido esférico cuyas condiciones de la masa son aquellas de haber estado inmersa por un tiempo infinito en un medio mantenido a temperatura constante, como lo puede ser el sol para la tierra después de un tiempo infinito, él define esta temperatura desde sus consideraciones preliminares como de valor uno, tal masa se expone después a otro medio como la puede ser el aire el cual define a temperatura cero, todo el sistema anterior está desplazándose con velocidad constante. Lo que quiere determinar son los estados sucesivos de la masa en el proceso de enfriamiento. Considera $\mathrm{x}$ la distancia de un

† Interpretación libre del capítulo II sección II, pp. 90-92. Para ver anexo consultar Morales (2003) 
punto cualquiera al centro de la esfera a una temperatura $\mathrm{v}$ después de un tiempo $\mathrm{t}$, además de que considera que $\mathrm{v}$ es diferente para valores diferentes de $\mathrm{x}$, el cual hubiera sido el caso si la inversión no durara un tiempo infinito.

Los puntos de un sólido, a iguales distancias del centro, tendrán una temperatura común; $\mathrm{v}$ es entonces una función de $\mathrm{x}$ y $\mathrm{t}$. Cuando suponemos $\mathrm{t}=0$, es esencial que el valor de esta función este de acuerdo con el estado inicial dado, y el cual es enteramente arbitrario.

Consideremos el movimiento que el calor sigue en un instante, y para una capa del sólido esférico infinitamente delgada y limitada por otras dos superficies esféricas con radios $\mathrm{x} \mathrm{y}(\mathrm{x}+\mathrm{dx})$ la cantidad de calor transferido a la primera capa, depende de cuatro factores a saber: la naturaleza de la cáscara representada por $\mathrm{K}$, el instante de tiempo t, el área de la cáscara y la proporción que no dice cómo varia la temperatura con respecto a la posición (ley de Fourier). Finalmente, ya que este calor está dejando a la cáscara esférica es tomado con signo negativo (ver figura 1):

$$
-4 K \pi x^{2} \frac{\partial v}{\partial x} d t
$$

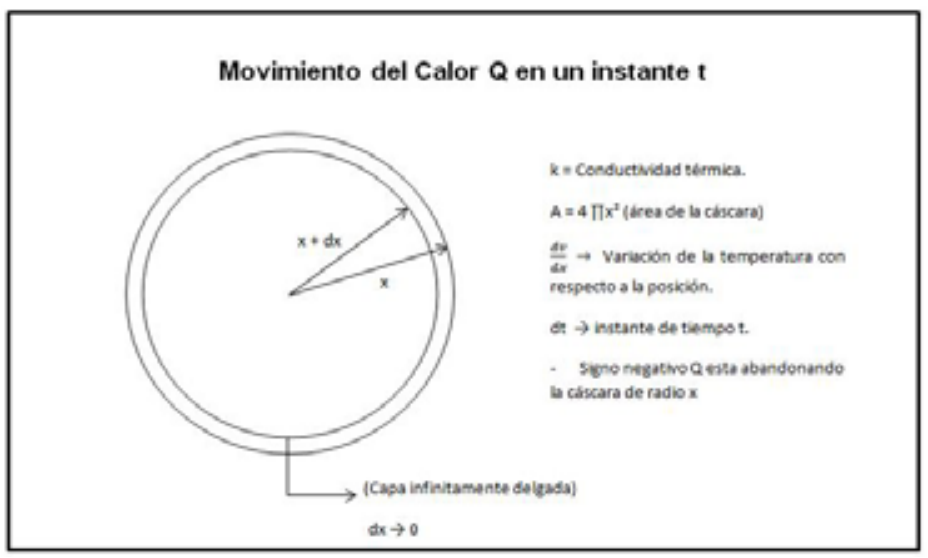

Figura 1.

De igual manera obtiene la cantidad de calor que deja a la segunda capa, la expresión es la misma que para la primera sólo se le adiciona el diferencial del término con respecto a $\mathrm{x}$. La diferencia entre el calor que entra a través de la primera capa y el que deja a la segunda nos dará el calor que se acumula en el interior de ambas y cuyo efecto consiste en variar la temperatura de la capa intermedia.

$$
-4 K \pi x^{2} \frac{\partial v}{\partial x} d t-4 K \pi \frac{\partial}{\partial x}\left(x^{2} \frac{\partial v}{\partial x}\right) d x d t
$$

\footnotetext{
* Conductividad térmica que depende del material del sólido en cuestión.
} 
Como la cantidad de calor que deja la capa esférica a través de la segunda superficie; y si sustraemos esta cantidad de aquella que entra a través de la primera superficie, tendremos:

$$
4 K \pi \frac{\partial}{\partial x}\left(x^{2} \frac{\partial v}{\partial x}\right) d x d t
$$

Esta diferencia es evidentemente la cantidad de calor que se acumula en la capa intermedia y cuyo efecto es variar su temperatura. El coeficiente $\mathrm{C}$ denota la cantidad de calor necesario para elevar desde cero hasta uno, un definida unidad de peso, La cantidad de calor necesario para elevar una unidad de peso $\mathrm{D}^{\S}$ desde una temperatura 0 hasta una temperatura 1 depende del material del que pudiera componerse la esfera, esta cantidad es la capacidad específica y es denotada por $C^{* *}$ el volumen de la capa intermedia según se ve arriba será $4 \pi \chi^{2} d x$, por tanto $4 \pi C D \chi^{2} d x$ será la cantidad de calor que se necesita para elevar la cáscara intermedia desde cero hasta uno, dividiendo esta cantidad entre el calor acumulado y derivando para obtener el aumento de la temperatura con respecto del tiempo encuentra la ecuación diferencial que representa el movimiento del calor al interior de la esfera:

$$
\begin{gathered}
d v=\frac{K}{C D} d t \frac{\mathrm{I}}{x^{2}} \frac{\partial}{\partial x}\left(x^{2} \frac{\partial v}{\partial x}\right) \\
\text { Ó } \\
\frac{\partial v}{\partial t}=\frac{K}{C D}\left(\frac{\partial^{2} v}{\partial x^{2}}+\frac{2}{x} \frac{\partial v}{\partial x}\right) \ldots \ldots \ldots . .
\end{gathered}
$$

Esta ecuación representa la ley que sigue el movimiento del calor en el interior de la esfera sólida. Fourier continua en su obra deduciendo las ecuaciones para otro tipo de sólidos, sin embargo regresa en especial a esta, en sus memorias de la real academia de ciencias del instituto de Francia, intituladas: Theorie du mouvement de la chaleur dans les corps sólides. Publicadas en 1826 donde explica el motivo de su investigación y en especial la aplicación de la ecuación de calor para una esfera sólida.

$\S$ Vemos que aquí $D$ se refiere a la densidad, en este caso volumétrica del sólido.

** La relación que existe entre le energía transferida a una sustancia y su temperatura se puede expresar en términos de la termodinámica como: $d Q=m C d T$, extra expresión índica como varía energía transferida con respecto a la temperatura, donde $m$ representa la unidad de masa y $\mathrm{C}$ la capacidad térmica por unidad de masa, es decir el calor específico, cuyas unidades consecuentes con el sistema son de energía por unidad de masa por unidad de temperatura [J/gr ${ }^{\circ} \mathrm{C}$ ]. Podemos ver entonces que el coeficiente $\mathrm{C}$ que Fourier define como capacidad específica es el calor específico y la forma en cómo está definido, lo que lo acerca más a la noción de calor en el sentido coloquial o de sustancia que hemos venido mencionando. 
Asevera que después de haber expuesto las leyes generales de movimiento de calor en cuerpos sólidos, resultará útil indicar una de las principales aplicaciones de su teoría, y que ha elegido para ese objetivo el asunto de las temperaturas terrestres ya que es un estudio que le interesa más que ningún otro, aunque el examen de este problema exigía muchas observaciones exactas y variadas no se podían determinar por la falta de una teoría, pero en vista de que él ya la estableció, tratará de recopilar todas las observaciones y hechos que pudieran ayudar.

De manera general se puede decir que en este documento Fourier se plantea dos problemas a solucionar:

1. Considerando las oscilaciones periódicas de calor que actúan sobre la superficie terrestre ¿Cuál es la temperatura de un punto dado inmediatamente por debajo de dicha superficie? a una distancia relativamente pequeña.

2. ¿Cuál es la temperatura fija en un punto dado más allá de la superficie, esto es en un punto que no participa de las oscilaciones periódicas de la superficie?

Para lograrlo cuenta con las siguientes observaciones y elementos:

Variabilidad. Los diferentes puntos de la superficie terrestre son irregularmente expuestos a la acción de los rayos solares, los movimientos de rotación y traslación de la tierra vuelven muy variables los efectos sucesivos del sol; si se colocaran termómetros en diferentes puntos de la parte sólida de la tierra, inmediatamente por debajo de la superficie, se observarían los cambios continuos en cada uno de los termómetros. Esos movimientos de calor en la superficie tendrán también influencia en todo lo que ocurra en el interior del planeta.

Periodicidad. Las grandes variaciones de la temperatura en la superficie del globo son periódicas, se reproducen y se repiten sensiblemente después del intervalo de un año. Así el asunto consiste principalmente en determinar el movimiento de calor en una esfera sólida con un diámetro considerado inmenso, cuya superficie está sometida a la acción periódica de un foco exterior.

Equilibrio. Si se supone que todos los puntos de la superficie de un globo sólido inmenso fueran sometidos, por una causa exterior cualquiera y durante un tiempo infinito, a los cambios periódicos de temperatura iguales a éstos que observamos, estas variaciones no podrían afectar más que una envoltura esférica cuyo grosor es infinitamente pequeño en relación con el radio; es decir, que en una profundidad vertical poco considerable, la temperatura de un punto tendrá un valor constante que depende, siguiendo una cierta ley, de todas las temperaturas variables de un punto de la misma vertical situada en la superficie. Pero, se debe observar que el valor fijo de la temperatura no es el mismo cuando se cambia de vertical, porque se supone que los puntos correspondientes de la superficie afectan irregularmente la acción 
del foco exterior. Si se hace caso omiso a la envoltura del globo sólido, se podrá decir que los diversos puntos de su superficie están sometidos a las temperaturas constantes por cada uno de esos puntos pero irregulares para los puntos diferentes. Y con la siguiente:

Hipótesis: Se supondrá entonces, en primer lugar, que la superficie de una esfera sólida, de gran diámetro, está sometida en sus diversos puntos a los cambios periódicos de temperatura, equivalentes a los que observamos hacia la superficie de la Tierra. Primero, considerara hacer caso omiso de los movimientos del calor en el sentido horizontal. En efecto, todos los puntos de la superficie que están contiguos, y comprendidos en una muy vasta extensión, deben ser vistos como igualmente afectados por las causas exteriores: resulta que los puntos correspondientes colocados en el interior a una profundidad poco considerable tienen además, en el mismo instante, temperaturas sensiblemente iguales; así pues se comunican las cantidades de calor extremadamente pequeñas. No es lo mismo en los puntos contiguos de una misma línea vertical; sus temperaturas, tomadas al mismo instante, difieren entre si en cantidades incomparablemente más grandes que aquellas de puntos igualmente distantes de la superficie. Por consiguiente, el movimiento de calor que se trata de conocer, por una línea vertical dada, es sensiblemente la misma que si todos los puntos de la superficie de la esfera sufrieran los cambios periódicos enteramente parecidos. Lo puntos igualmente distantes del centro de la esfera conservan entonces una temperatura común v que varía con el tiempo transcurrido t. Designando por $\mathrm{x}$ la distancia al centro, se ve que $\mathrm{v}$ es una función de $\mathrm{x}$ y $\mathrm{t}$ que se debe determinar.

Así la ecuación:

$$
\frac{\partial v}{\partial t}=\frac{K}{C D}\left(\frac{\partial^{2} v}{\partial x^{2}}+\frac{2}{x} \frac{\partial v}{\partial x}\right)
$$

Que se ha obtenido anteriormente representa las variaciones instantáneas de temperaturas en una esfera sólida en la cual las capas esféricas son irregularmente calentadas; es decir que si actualmente se le diera a los puntos de la esfera situados a la distancia $\mathrm{x}$ una temperatura $\mathrm{v}, \mathrm{v}$ siendo una función de $\mathrm{x}$ dada, $\mathrm{y}$ que se quisiera conocer el resultado instantáneo de la acción mutua de todas las partículas, se debería unir a la temperatura de cada punto la diferencial de tiempo

$$
\frac{K}{C D}\left(\frac{\partial^{2} v}{\partial x^{2}}+\frac{2}{x} \frac{\partial v}{\partial x}\right) d t
$$

En el asunto que se trata ahora, se reemplazará la variable $\mathrm{x}$ por $\mathrm{X}-\mathrm{u}, \mathrm{X}$ representa el radio total de la esfera, $\mathrm{y} u$ la distancia perpendicular entre la superficie y el punto 
del cual la temperatura es v. Se obtiene por esta sustitución, y considerando X como un número muy grande tenemos

$$
\frac{\partial v}{\partial t}=\frac{K}{C D}\left(\frac{\partial^{2} v}{\partial u^{2}}\right)
$$

El resultado que presenta esta ecuación es coherente con la hipótesis presentada inicialmente por Fourier, y con la pregunta que se está respondiendo; ya que recordaremos, desea conocer la temperatura de un punto dado inmediatamente por debajo de la superficie, a una "temperatura mediocre" como él la llama, de esta manera u representará una distancia pequeña y por tanto $\mathrm{X}-\mathrm{u}$ un valor muy grande, dando como resultado (2) la cual por cierto, introduce una posición más general del punto a observar, es decir, que en la última expresión u en realidad también es $\mathrm{u}(\mathrm{x}, \mathrm{y}, \mathrm{z})$.

En la actualidad a la ecuación diferencial (2) en general se le conoce como la ecuación de difusión de calor en estado transitorio o no estacionario, con algunas pequeñas variaciones en la notación:

$$
\nabla^{2} V=\left(\frac{1}{K}\right)\left(\frac{\partial V}{\partial t}\right)
$$

Dónde:

$$
\nabla^{2}=\frac{\partial^{2}}{\partial^{2} x}+\frac{\partial^{2}}{\partial^{2} y}+\frac{\partial^{2}}{\partial^{2} z}
$$

$\mathrm{Y}$ donde $\mathrm{K}=\mathrm{k} / \mathrm{\rho C}$ llamada constante de difusividad o simplemente difusividad ${ }^{\dagger \dagger}$ , con $\mathrm{k}$ la conductividad propiamente mencionada que representa la naturaleza del sólido, $\rho$ la densidad volumétrica y $\mathrm{C}$ el calor específico.

\section{Solución de la ecuación de difusión de calor o el "problema de Fourier"}

Por último, revisaremos la solución de la mencionada ecuación en la forma deducida por Fourier haciendo a su vez uso de los elementos e hipótesis planteadas al inicio del documento y que se traducen en esta sección como condiciones de frontera, cabe mencionar que el método de solución que él provee para determinar la distribución de temperaturas, representó en su tiempo un método matemático novedoso y que se usa hasta la actualidad, es de hecho por la solución completa

${ }^{\dagger+}$ Fue nombrada difusividad térmica por Lord Kelvin (Unsworth \& Duarte, 1979) 
de este problema que Fourier tiene su máximo reconocimiento entre la comunidad matemática, y por el cual algunos autores lo denominan "el problema de Fourier".

En la ecuación (2) y de la deducción se observa que $\mathrm{v}$ representa la función de la distribución de temperaturas, $t$ representa la unidad de tiempo y u (aún en el caso más generalizado) representa el radio de la posición del punto en la esfera, de esta manera y reescribimos la ecuación como:

$$
\frac{\partial^{2} V}{\partial r^{2}}=\frac{1}{k} \frac{\partial V}{\partial t}
$$

Suponiendo por el método de separación de variables, que la solución de (3) es de la forma:

$$
\begin{gathered}
\mathrm{V}(\mathrm{r}, \mathrm{t})=\mathrm{R}(\mathrm{r}) \mathrm{T}(\mathrm{t}) \quad \ldots \ldots .(4) \text { entonces } \frac{\partial V}{\partial t}=R(r) T^{\prime}(t) \\
\frac{\partial^{2} V}{\partial r^{2}}=T(t) R^{\prime \prime}(r) \ldots \ldots \ldots \ldots(6)
\end{gathered}
$$

Sustituyendo (5) y (6) en (3)

$$
R^{\prime \prime}(r) T(t)=\frac{1}{k} R(r) T^{\prime \prime}(t)
$$

O bien

$$
\frac{R^{\prime \prime}}{R}=\frac{1}{K} \frac{T^{\prime}}{T}=-\lambda^{2}
$$

Entonces:

$$
\begin{gathered}
T^{\prime}+K \lambda^{2} T=0 \ldots \\
R^{\prime \prime}+\lambda^{2} R=0 .
\end{gathered}
$$

Solucionando (7)

$$
\begin{aligned}
& \frac{1}{k \lambda^{2}} \ln T=C_{1} t \\
& T(t)=C_{1} e^{-\lambda^{2} k t}
\end{aligned}
$$

Solucionando (8) una ecuación diferencial homogénea de segundo orden, cuya ecuación característica tiene raíces imaginarias tenemos

$$
R(r)=C_{2} \cos \lambda r+C_{3} \operatorname{sen} \lambda r
$$

\footnotetext{
\$ Método de separación de variables.
} 
Sustituyendo (9) y (10) en (4)

$$
V(r, t)=C_{1} e^{-\lambda^{2} k t}\left[C_{2} \cos \lambda r+C_{3} \operatorname{sen} \lambda r\right] .
$$

El valor de las constantes en esta solución dependerá siempre de las condiciones iniciales y de frontera del problema, en este caso retomaremos aquellas que Fourier establece para el caso de la esfera calentada y sumergida en un medio a temperatura Ts para enfriarse paulatinamente. Asumimos así que Ts $=0$ y cuando $r=0$ y $r=a$, (con a el radio total de la esfera calentada) la distribución $\mathrm{V}=0$ esto es, $\mathrm{V}(0, \mathrm{t})=0$ y $\mathrm{V}(\mathrm{a}, \mathrm{t})=0$, además en $\mathrm{t}=0$ para un punto cualquiera de la esfera, es decir, un punto en la región $0 \leq \mathrm{r} \leq \mathrm{a} \rightarrow \mathrm{V}(\mathrm{r}, 0)=\mathrm{rT} 0$.

$\Rightarrow$

$$
T(0)=C_{1} e^{-\lambda^{2} k t} \text { Cuando t }=0 \quad \Rightarrow C_{1}=T_{0}
$$

También

$$
\begin{gathered}
R(0)=C_{2} \cos \lambda(0)+C_{3} \operatorname{sen} \lambda(0)=0 \\
C_{2} \cos (0)=0 \quad \therefore \quad C_{2}=0 \\
R(r)=C_{3} \operatorname{sen} \lambda r
\end{gathered}
$$

Reescribiendo (11)

$$
V(r, t)=e^{-\lambda^{2} k t} T_{0} C_{3} \operatorname{sen} \lambda r
$$

Por el principio de superposición,

Además,

$$
V(r, t)=T_{0} \sum_{n=1}^{\infty} C_{3} e^{-\lambda^{2} k t} \operatorname{sen} \lambda r
$$

$$
R(a)=C_{3} \cos \lambda a=0
$$

La constante es diferente de cero ya que de otra manera encontraríamos la solución trivial, en ese caso queda:

$$
\operatorname{sen} \lambda \mathrm{a}=0
$$

Y para que esta condición se cumpla

$$
\lambda a=n \pi \Rightarrow \lambda=\frac{n \pi}{a}
$$

Sustituyendo en (13)

$$
\text { Con } n=0, \pm 1, \pm 2, \pm 3, \ldots \ldots
$$

$$
V(r, t)=T_{0} \sum_{n=1}^{\infty} C_{3} e^{-\frac{k n^{2} \pi^{2}}{a^{2}} t} \operatorname{sen} \frac{n \pi}{a} r
$$


La última condición $\mathrm{V}(\mathrm{r}, 0)=\mathrm{rT} 0$ propone que:

$$
V(r, 0)=T_{0} \sum_{n=1}^{\infty} C_{3} e^{-\frac{k n^{2} \pi^{2}}{a^{2}}(0)} \operatorname{sen} \frac{n \pi}{a} r=\mathrm{rT}_{0}
$$

Simplificando

$$
\sum_{n=1}^{\infty} C_{3} \operatorname{sen} \frac{n \pi}{a} r=\mathrm{r}
$$

Los coeficientes C3, llamados coeficientes de Fourier se encuentran resolviendo la siguiente forma ${ }^{\S}$

$$
C_{3}=\frac{2}{a} \int_{0}^{a} r \operatorname{sen} \frac{n \pi}{a} r d r
$$

Solución:

$$
\begin{gathered}
C_{3}=\frac{2}{a}\left[-\frac{a r}{n \pi} \cos \frac{n \pi}{a} r+\frac{a}{n \pi} \int \cos \frac{n \pi}{a} r d r\right] \\
=\frac{a}{2}\left[-\frac{a r}{n \pi} \cos \frac{n \pi}{a} r+\frac{a^{2}}{n^{2} \pi^{2}} \operatorname{sen} \frac{n \pi}{a} r\right]_{0}^{a} \\
=\frac{2}{a}\left[-\frac{a^{2}}{n \pi} \cos n \pi\right] \\
=-\frac{2 a}{n \pi} \cos n \pi
\end{gathered}
$$

O bien:

$$
C_{3}=\frac{2 a(-1)^{n+1}}{n \pi}
$$

Sustituyendo los coeficientes de Fourier en (14) obtenemos la solución completa del problema de transferencia de calor por conducción

$$
V(r, t)=T_{0} \sum_{n=1}^{\infty} \frac{2 a(-1)^{n+1}}{n \pi} e^{-\frac{k n^{2} \pi^{2}}{a^{2}} t} \operatorname{sen} \frac{n \pi}{a} r
$$

$\S \S$ "Si se requiere obtener la expansión de una función dada $f(x),-L<x<L$, en una serie de Fourier de modo que: $f(x)=a_{f} / 2+\sum_{(n=1)}^{\infty} \square\left(a_{n} \cos n \pi x / L+b_{n} \operatorname{sen} n \pi x / L\right)$ entonces los coeficientes de Fourier estarán dados por $a_{n}=1 / L \int_{-L}^{L} f(x) \cos n \pi x / L d x, b_{n}=1 / L \int_{-L}^{L} f(x) \operatorname{sen} n \pi x / L$. Para el termino seno, la función $f(x)$ es una función impar, esto es, $f(-x)=-f(x)$ y los coeficientes se pueden expresar como: $a_{n}=0$ y $b_{n}=2 / L \int_{L}^{0} f(x) \operatorname{sen} n \pi x / L d x " \ldots \ldots$ (Spiegel \& Murray, 1983) 
La expresión (15) representa la distribución de temperaturas en un punto dado de una esfera sólida cómo lo puede ser la tierra dadas las condiciones de haber estado por mucho tiempo sometida a los efectos del sol y después haberse enfriado debido al contacto con el aire o medio ambiente.

Los primeros significados que se rescatan en el entendimiento del problema de calentamiento de la tierra desde la perspectiva de Fourier, son no sólo el establecimiento de las hipótesis del problema físico sino las conclusiones que se desprenden del desarrollo como la dependiente con el gradiente de temperaturas y con las características internas del sólido; más precisamente con la conductividad térmica; otro aspecto sutil pero igualmente importante es el concepto de capacidad calorífica, que pone de manifiesto que la conducción de calor proviene de una resistencia que opone un cuerpo a ser calentado y su disponibilidad para conducir el calor. Ya que Fourier se permite prever desde su teoría del calor las aplicaciones para las cuales está estaba dirigida, resalta por su importancia el concepto de flujo de calor y la conceptualización consecuente de que sin un conocimiento preciso de la expresión matemática para este flujo como función de la temperatura, el problema del calentamiento terrestre no sería posible de abordar.

\section{Implicaciones de la investigación}

La modelación matemática es un tema con muy diversas e importantes aplicaciones, prueba de ello son las aportaciones y trabajos que muchos colegas de nuestra disciplina han realizado y dedicado a ello, pero también las múltiples perspectivas desde las que esta actividad ha sido observada. La experiencia como profesores nos provee de la certeza de que el discurso matemático escolar se compone en su mayoría de las notas y de los libros de texto que se recomiende usar para apoyar las actividades y cubrir el programa. Sin embargo en este saber no están incorporados los significados primarios, ya que se ignoran las etapas, objetivos, momentos y contextos por los que ha transitado el conocimiento desde su construcción original. Es aquí donde un estudio de corte epistemológico podría proveer de argumentos olvidados por la enseñanza tradicional, de forma tal que a través de este tipo de estudios sea posible dar enfoques diferentes o alternativos a los conceptos estudiados en el nivel superior. El tipo de análisis de un fenómeno como el que nos ocupa, busca establecer un acercamiento a la génesis y desarrollo de los conceptos que en este se establecen, con el objeto de encontrar en él los elementos que nos permitan obtener un mejor entendimiento de las características necesarias a la actividad matemática.

En el marco de la didáctica fundamental, se pone de manifiesto que no es posible interpretar la matemática escolar ni la actividad matemática, sin tener en cuenta los fenómenos relacionados con la reconstrucción de la matemáticas que tienen su origen en la propia institución de producción del saber matemático (Chevallard, 
1991). El desarrollo de esta teoría ha aportado argumentos para justificar por qué no pueden separarse completamente el estudio de la génesis y el desarrollo del saber matemático, del estudio de la enseñanza y la utilización de dicho saber.

$\mathrm{Y}$ es por estos mismos argumentos que se considera necesario, adoptar una perspectiva que incluya una visión, sensible al reconocimiento de que el conocimiento es una construcción social. Así las teorías frutos o consecuencias de las líneas de investigación sostenidas por la comunidad de especialistas en matemática educativa, tal como la aproximación socioepistemológica ${ }^{* * *}$, perspectiva que hace dicho énfasis en la naturaleza social de la actividad de la construcción por parte de los actores sociales en contextos sociales concretos, más específicamente, la socioepistemología, plantea el examen del conocimiento social, histórica y culturalmente situado, problematizando a la luz de las circunstancias de su construcción y difusión (Cordero, 1999). Coincidimos con Arrieta (2003) al asegurar desde esta posición, que lo socioepistemológico debe significar, el reflejo de cualquier actividad humana haciendo matemáticas.

De esta forma a la actividad de modelación y al uso de las matemáticas dentro de la misma le es inherente un proceso mental que está estrechamente relacionado e influido por su entorno sociocultural, en este caso por la época en la que Fourier y sus escritos surgen. Fourier se preguntaba acerca de un fenómeno físico observable y que le generaba preguntas que para la época era difícil contestar; sin embargo él se da a la tarea de desarrollar todo el constructo teórico (matemático) que le permita obtener respuestas, tal desarrollo está íntimamente relacionado con el contexto histórico y entorno de la época cuyas limitaciones matemáticas, científicas y de desarrollo tecnológico marcan el modelo matemático que el propio Fourier establece. El punto que se remarca en este escrito y que es propuesto por la visión socioepistemológica, es aquel de decir que con otras herramientas didácticas, tecnológicas y científicas como las que actualmente poseemos; se generarían a partir de la misma pregunta inicial, un constructo o conocimiento completamente diferente, cuya aceptación y entendimiento por la comunidad didáctica (institución educativa) dependería en mucho de incluir también el contexto que le dio vida. O como lo afirma la teoría: no puede separarse completamente el estudio de la génesis y desarrollo del saber matemático, del estudio, de la enseñanza y la utilización de dicho saber. Cuestión que por lo demás a llevado a la búsqueda de diferentes enfoques didácticos que permitan la inclusión de la componente social, epistemológica, cognitiva y didáctica.

Así el tipo de investigación que llevamos a cabo, visto como la investigación encaminada a la reconstrucción de significados, nos permite rescatar de los trabajos de Fourier algunos significados o intenciones primarias de los conceptos matemáticos que él generó, a través de cuestionarnos cómo y atendiendo a qué problema surgieron estos.

"*Desarrollada por el grupo del Área de Educación Superior del Departamento de Matemática Educativa del CINVESTAV, IPN. 


\section{Referencias Bibliográficas}

-Albert A. (1999). Introducción a la Epistemología. Serie: Antologías 2, 1-28

-Arrieta J. (2003). La modelación de fenómenos como procesos de matematización en el aula. Tesis Doctoral (Inédita), Cinvestav México.

-Cantoral y Farfán (2003). Matemática educativa: Una visión de su evolución, Revista Latinoamericana de investigación en Matemática Educativa, Vol.6, núm. 1, Marzo, 2003, pp. 27-40.

-Cordero F. (1999). El "comportamiento tendencial de las funciones" como una categoría del conocimiento del Cálculo. Serie Antologías 2, 29-54.

-Chevallard Y. (1991). La transposición didáctica. Del saber sabio al saber enseñado. Buenos Aires Aique (C. Gilman Trad)

-Fárfan R. (1997). Ingeniería didáctica: Un estudio de la variación y el Cambio. Grupo Editorial Iberoamerica, S.A. de C.V. Méx.

-Farfán R. M. (1999). Perspectivas y Métodos de investigación en Matemática Educativa. Serie Antologías 2, 55-120

-Fourier J.B. (1827). Theorie Analytique de la Chaleur. Asociación Mexicana Clásicos de la Ciencia. México, 1963.

-Fourier J. B. J. (1827). Mémoires d'l académie Royale des sciences de l'Institute de France VII, 570-604. París, Didot.

-Morales A. F. (2003). Acerca de la actividad de modelación. Las temperaturas de la Tierra. Tesis de maestría no publicada. Cinvestav IPN, México.

-Spiegel M. R. (1983). Ecuaciones diferenciales aplicadas. Prentice-Hall Hispanoamericana, S.A. México.

-Unsworth J and Duarte F. (1979). Heat diffusion in a solid sphere and Fourier theory: an elementary practical example Am. J. Phys. 47 981-3. 